\title{
LIBROS SOBRE PSICOPEDAGOGÍA
}

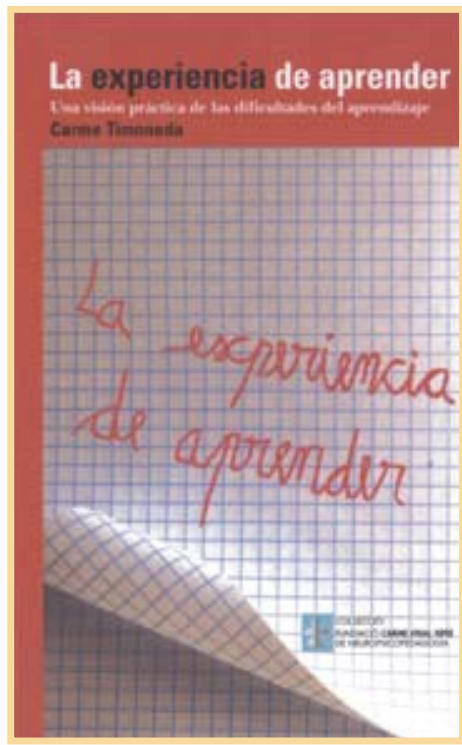
aprender: una visión práctica de las dificultades del aprendizaje.

Timoneda Gayart, C. CCG Edicions, 2006

Girona. 94 págs.

rase una vez una abuela y su nieta que Econtemplaban las llamas del fuego; cada atardecer se sentaban una al lado de la otra, en la calidez del hogar, y la nieta escuchaba embobada las historias de su abuela. Las recordaba todas, palabra por palabra... Aún ahora, treinta años más tarde, cuando ya no tiene a la abuela a su lado, puede sentarse al lado del mismo
La experiencia de

fuego y recordar todas y cada una de las historias... ¡Qué añoranza siente! En cambio, no añora tanto la escuela... ¡No recuerda demasiados buenos momentos! Allí no le explicaban historias... La historia que ella estudiaba era aburrida y pesada..., nunca se acordaba de gran cosa, y le resuenan aún las palabras de aquella señorita: «¡Qué memoria tan pobre que tienes!» ¡Y qué sacrificio era para ella ponerse a estudiar e intentar recordar todo lo que estaba escrito en los libros! ¡Y las notas! ¡Qué suplicio! Gritos de los padres, represalias de los maestros... Éstos son los recuerdos amargos que le vienen a la cabeza suavizados por la dulzura del recuerdo de las horas pasadas con la abuela al lado del fuego. La abuela no le gritaba..., la escuchaba..., siempre tenía unas palabras que la hacían pensar pero nunca le decía lo que estaba bien y lo que no y siempre tenia a punto una historia para explicar.

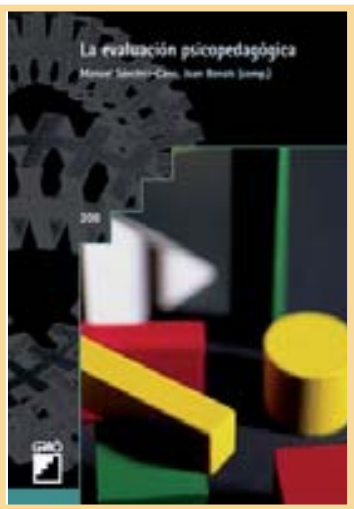

\section{La evaluación}

\section{psicopedagógica}

Sánchez-Cano, M., y Bonals, J. (COMP.)

Graó, 2007

Barcelona. 456 págs.

neurología o la computación como ocurre en otros casos. En segundo lugar, porque dichos conocimientos orbitan sobre el eje consciencia-inconsciente que, siendo fundamental, ha sido durante mucho tiempo dejado de lado por su complejidad.Y, en tercer lugar, porque está escrito para poder afrontar conjuntamente los grandes debates sobre la naturaleza de la consciencia, qué nos diferencia de las máquinas inteligentes, o si queda margen para la soñada libertad.

El libro está escrito de principio a fin en forma de diálogo para tratar de facilitar su lectura, si bien sus protagonistas no son Sócrates ni Platón, sino un profesor y un alumno de cualquier universidad española, eso sí, con una relación muy especial.
En esta obra, los autores aportan un Erecorrido actual y dinámico sobre la evaluación psicopedagógica. Partiendo de la propia evolución del sentido de la Evaluación psicopedagógica, ofrecen una perspectiva que considera el contexto en el que se desarrolla el alumno como elemento clave en el que encuadrar la acción evaluadora, tanto por lo que aporta al propio individuo como por lo que éste influye en el contexto. Desde esta perspectiva, el libro recorre el papel de los diferentes agentes educadores en la evaluación psicopedagógica, identificando dos grandes bloques temáticos: un primer apartado en el que los autores se centran en el concepto y elementos fundamentales de la evaluación psicopedagógica, y un segundo gran apartado en el que se abordan los aspectos relacionados con la evaluación psicopedagógica ante diferentes necesidades educativas específicas. 


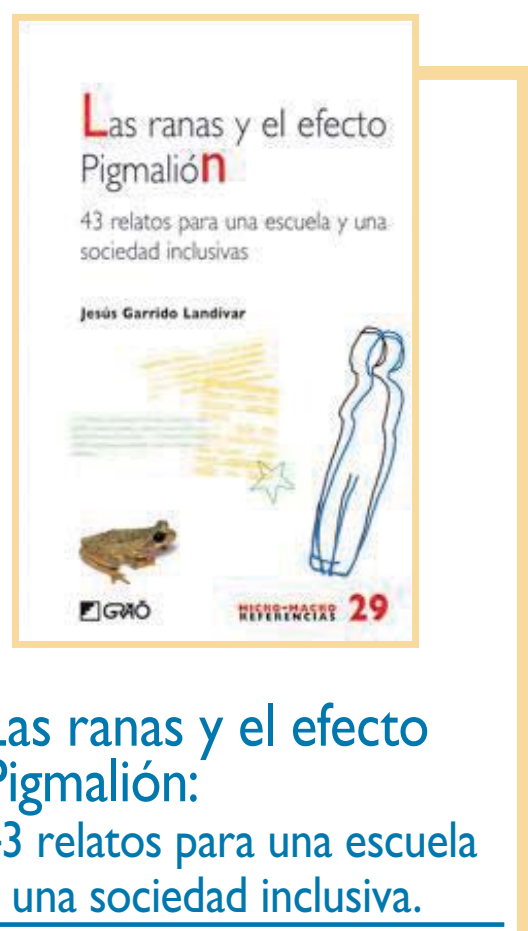

Garrido Landivar, J.

Graó, 2010

Barcelona. 179 págs.

En este libro,el autor, Catedrático de eduEcación especial de la Facultad de Formación del Profesorado de la Universidad de las Palmas de Gran Canaria (ULPGC) presenta un conjunto de historias breves, recopiladas de su dilatada experiencia en educación así como de relaciones en diferentes ámbitos, todas ellas encaminadas a ofrecer al lector perspectivas y miradas inclusivas en educación. La obra se dirige a padres y madres, a psicopedagogos, a los maestros, a todos aquellos que son agentes educadores. Estos relatos breves ofrecen una mirada tierna e inteligente sobre las diferentes realidades de discapacidad y necesidades específicas en educación y en la sociedad.

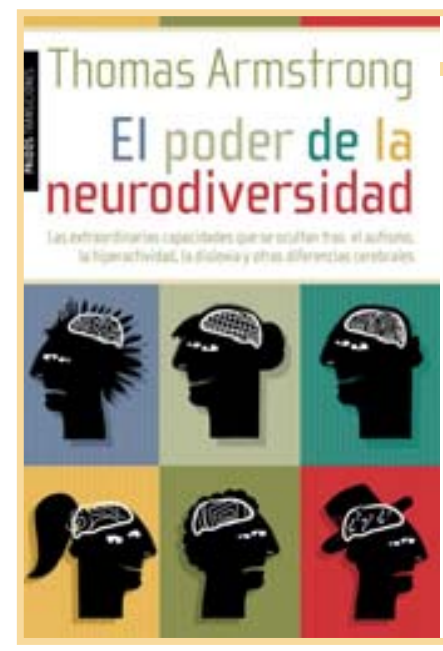

El poder de la neurodiversidad.

Las extraordinarias capacidades que se ocultan tras el autismo, la hiperactividad, la dislexia y otras diferencias cerebrales

Armstrong, T.

Paidós, 2012

Barcelona. 320 págs.

En esta obra, el autor presenta un conEcepto novedoso, el de "neurodiversidad", que se refiere a las capacidades diferentes y sorprendentes que presentan las personas con diagnósticos de diversas discapacidades en cuanto a ciertas capacidades mentales. Todo ello lleva a un concepto de etiqueta diagnóstica necesariamente relativo, en función del contexto cultural.A partir de esta exposición, el autor propone formas de aprovechamiento de las tecnologías de apoyo terapéutico para resaltar estas características propias de la neurodiversidad, así como una reflexión sobre el desarrollo profesional adulto de personas con neurodiversidad. Ofrece por tanto una mirada positiva sobre las diferencias neuropsicológicas de personas con diversos diagnósticos de necesidades educativas específicas.

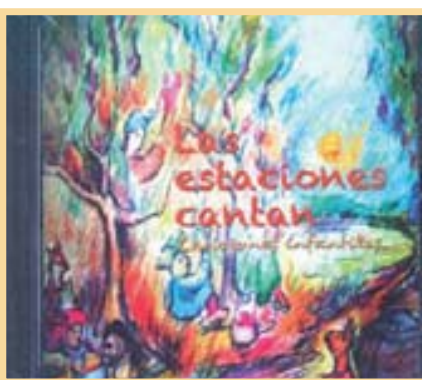

\section{Las estaciones cantan. Canciones infantiles.}

Álvarez Puerto, $C$.

Mitabom, 2009

Madrid.

Este CD presenta ocho canciones infanEtiles, cuatro para Infantil y cuatro para Primaria, que toman como excusa las cuatro estaciones para recrear los colores de la vida en sus múltiples tonalidades. Cristina Álvarez Puerto, con música de José Luis Merlín a la guitarra e ilustraciones de Helmut Mally, compone una sinfonía en la que se escucha la melodía de la naturaleza, el paso del tiempo y la vida que brota en cada niño. Algo se mueve algo se agita, El cole se acabó, Los vestidos del otoño, La luz del invierno, Fiesta de primavera, Ven conmigo a jugar y soñar, Los enanitos de otoño y Mi luz tu luz, los títulos de las canciones son invitaciones a adentrarnos en el rincón poético y musical tal vez perdido u olvidado de nuestra infancia y también a vivenciar la estacionalidad y las cualidades anímicas de cada estación del año. En los textos puede apreciarse el amplio bagaje pedagógico de la autora (www.cristinalvarezpuerto.es), su sensibilidad poética y también su deseo de conectarnos emocionalmente con lo mejor del ser humano, como la generosidad, el coraje o la alegría. Los educadores de Infantil y Primaria encontrarán aquí un valioso aliado en su tarea de todos los días.

\section{Próximos números}

\section{Diciembre}

\section{LA COMPETENCIA ESPIRITUAL}

La iniciación a la experiencia de Dios en niños.

Inteligencia espiritual por descubrimiento.

Coaching: una oportunidad para el desarrollo de la competencia espiritual.

Competencia espiritual y clases de religión.

\section{SECCIONES}

Música y competencias básicas.

Grandes de la educación.

Filmoteca Padres y Maestros.

Experiencias de innovación.

Una mirada sobre la educación.

Biblioteca PyM.

Febrero

EDUCACIÓN BILINGÜE EN INGLÉS 